\title{
An Experimental Study on the Acoustic Features of Vowels in Western Yugur Language
}

\author{
Fan Bai ${ }^{1, *}$, Shiliang $\mathrm{Lyu}^{2}$, Lin $\mathrm{Na}^{3}$ \\ ${ }^{1}$ Key Laboratory of China's Ethnic Languages and Information Technology of Ministry of Education, Northwest \\ Minzu University, Lanzhou, Gansu, China \\ ${ }^{2}$ Key Laboratory of China's Ethnic Languages and Information Technology of Ministry of Education, Northwest \\ Minzu University, Lanzhou, Gansu, China \\ ${ }^{3}$ Key Laboratory of China's Ethnic Languages and Information Technology of Ministry of Education, Northwest \\ Minzu University, Lanzhou, Gansu, China \\ *bf18893254226@126.com
}

\begin{abstract}
This article will use the theory of experimental phonetics to describe the acoustic characteristics of vowels in Western Yugur language, making the speech signal more specific and objective. Using the vowel distribution pattern theory and the vowel formant pattern, extract and analyze various acoustic parameters of vowels in Western Yugur language, establish acoustic vowel maps and vowel pattern maps, and explore the acoustic characteristics of vowels in Western Yugur language.
\end{abstract}

Keywords: Western Yugur, vowel pattern, vowel acoustic space, formant pattern

\section{INTRODUCTION}

The Yugur nationality is a minority ethnic group with a relatively small population in our country, and it is also a unique ethnic group in Gansu Province. According to statistics from the 2021 census, the number of Yugur people in China is 14,706. In addition to Chinese, local people also use Eastern Yugur and Western Yugur. ${ }^{[1]}$ The Eastern and Western Yugur languages belong to the Altaic language family, but they belong to different language families. The research on Western Yugur language mainly focuses on the classification and arrangement of vocabulary, or the comparative analysis of Western Yugur language and other minority languages. ${ }^{[2]}$ However, it lacks the use of acoustic experimental methods to make speech signal parameters concrete, qualitative, quantitative, objective, scientific, and accurate. This article will focus on the acoustic characteristics of vowels in Western Yugur language.

\section{EXPERIMENTAL DESIGN AND METHODS}

\subsection{Experimental equipment}

The recording equipment is a Lenovo notebook computer, a mixer, an external sound card, and a lavalier microphone. The software equipment has Audition3.0 recording software, each syllable is read twice, Praat6.0, SPSS software, etc.

\subsection{Subjects}

The selection of the corpus is mainly based on Lei Xuanchun's "Western Yugur Chinese Dictionary" and Chen Zongzhen's "Western Yugur Language Research". There are 8 basic vowels in Western Yugur: [a],[e],[ə],[i],[o],[u],[ø],[y]. ${ }^{[3]}$ And then classify these 8 vowels according to the vowels at the beginning of the word, the vowels at the end of the word, and the vowels at the end, and sort them in the excel table. The recording corpus has a total of 842 multi-syllable words, which basically cover the collocation of vowels and consonants in Western Yugur language. The corpus used in this chapter only takes female voice samples.

\subsection{Voice annotation}

Both speech annotation and extraction of acoustic parameters rely on Praat phonetics software. The software was originally named Praat: doing phonetics by computer, usually referred to as Praat, is a cross-platform multifunctional phonetics professional 
software, mainly used to analyze, label, process and synthesize digital speech signals, and generate various Sonograms and text reports.

\subsection{Parameter extraction}

When making the vowel pattern, the script "Drawing a vowel ellipse based on formant data" written by Ling Feng (Shanghai University) was used. ${ }^{[4]}$ By running the script with the extracted formant data, the vowel distribution pattern of Western Yugur language can be obtained. picture. The praat software developed by Paul Boersma \& David Weenink (2020) and modified by Bei Xianming and Xiang Ning (2020) in Chinese, uses this software to extract the first formant (F1), second formant (F2), and first formant of vowels. Three formants (F3), through the data of three formants, you can get the $\mathrm{V}$-value graph and $\mathrm{V}$-value table of vowels, and draw the sound position graph and acoustic vowel scatter diagram of vowels.

\section{THE DISTRIBUTION PATTERN OF VOWELS IN WESTERN YUGUR LANGUAGE}

The view of phonetic pattern was put forward by Mr. Shi Feng, whose aim is that every language and dialect is systematic, and the phonetic pattern is the expression of the systemic phonetics. ${ }^{[5]}$ The vowel phoneme map of Western Yugur language obtained through the vowel pattern experiment reflects the acoustic spatial distribution and vowel pattern of vowels in Western Yugur language. The frequency of the same vowel formant produced by different speakers is different, but the relative position of each vowel produced by each person on the acoustic vowel map is basically stable. The following is the spatial distribution map of vowels in Western Yugur language.

\subsection{Acoustic vowel diagram}

The ellipse diagram of the distribution of vowels in Western Yugur language can roughly show the distribution pattern of eight vowels, and intuitively see the distribution characteristics of vowels and vowels that are related or opposed. According to the parameters of the initial vowel formants (F1, F2), run the script that draws the vowel ellipse, and you can get the distribution ellipse of the initial vowel, the middle vowel, and the final vowel respectively. Each ellipse represents the distribution range of each vowel. Figure 1,2, and 3 respectively show the acoustic distribution patterns of the initial vowels, middle vowels, and final vowels of a word. Since the vowel [ø] does not appear at the end of a word in Western Yugur, only seven vowels appear in the ellipse of ending vowels. The details are shown in the figure below.

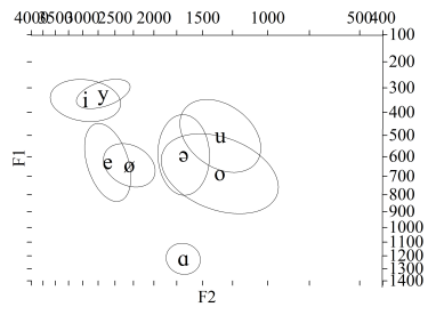

Figure 1 Oval diagram of the initial vowel of a word

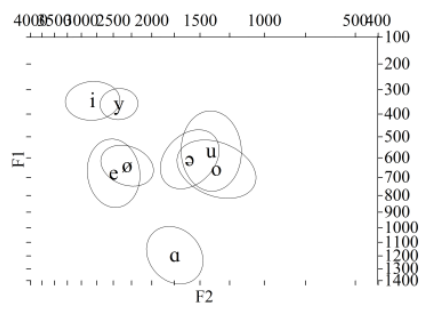

Figure 2 Oval Diagram of Vowels in Words

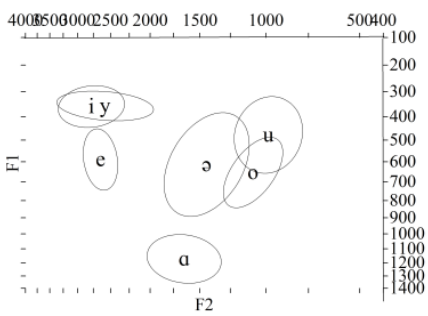

Figure 3 Oval diagram of ending vowels

Since [ø] does not appear at the end of a word in Western Yugur, only seven vowels appear in the ellipse of ending vowels. From the ellipse diagram of the ending vowel, it can be seen that [i] and [y] are at the apex position, [i] and [y] have the largest F2 and the smallest F1. According to the distribution of the vowel formant and the tongue position of the vowel Relation, it can be concluded that [i] and [y] are a high vowel, [y] is a bit more forward than [i], and [y] is a bit higher than [i]. [a] The largest F1 indicates the lowest tongue position. The lowest $\mathrm{F} 2$ of [o] means that the tongue is the most rearward.

Conclusion: From the overall view of the three acoustic vowel diagrams of the beginning, the middle and the end of the word, the distribution of the ending vowels is more scattered than that of the beginning and the middle of the word. The distribution range of the ending vowel F2 is roughly $3500 \mathrm{~Hz} \sim 600 \mathrm{~Hz}$, and the distribution range of the vowel F2 at the beginning and middle of the word is roughly $3500 \mathrm{~Hz} \sim 1000 \mathrm{~Hz}$, which shows that the lateral span of the ending vowel is relatively large. Now we analyze the distribution of each vowel map in detail.

(1) From the perspective of the high and low dimensions of vowels, it is mainly determined by the first formant (F1). [a] No matter it is at the beginning, middle and end of the word, it is in the highest position. 
The value of F1 is the largest, indicating that [a] When the tongue is the lowest, it is a low vowel. [i] and [y] are both at the lowest, and the F1 value is the smallest, indicating that [i] and [y] are pronounced with the highest tongue position and are high vowels.

(2) From the perspective of the front and back dimensions of the vowel, it is mainly determined by the second formant (F2). No matter which position [i] is in, the value is the largest, which also shows that the tongue position of [i] is at the forefront The position of, belongs to the front vowel. [y] is close to [i] in the three figures, so it can be seen that [y] also belongs to the front vowel. Secondly, the F2 values of [o] and [u] are relatively small compared to other vowels. It can be seen that the tongue positions of $[\mathrm{o}]$ and $[\mathrm{u}]$ are relatively backward.

(3) From the three vowel ellipse diagrams, there are some overlapping ellipses. $[\mathrm{o}],[\mathrm{u}]$ and [ə], the vowels are more concentrated in the beginning of the word and in the word. There are also [i] and [y], [ø] and [e], indicating that these vowels are pronounced similarly in some syllables, and that there are some phonemic variants in the pronunciation of these vowels.

\subsection{Vowel pattern}

Use Praat software to extract the first three formants (F1, F2, F3) of the middle vowel phoneme at the beginning of each word, and run the software program to get the vowel pattern, and put the tongue in the top position in the picture. The three vowels with the lowest tongue position and the largest opening are connected to form the vowel pattern of Western Yugur language. The vowel pattern map can reflect the relative positional relationship between the apex vowels and non-apex vowels in Western Yugur language.

The following is also presented in the form of vowels at the beginning, middle, and end of a word respectively. Since [ø] does not appear at the end of a word, only seven vowels are shown in the pattern of ending vowels. The details are shown in Figure 2.4, 2.5, and 2.6. During the experiment, the $\mathrm{V}$ value table of each vowel can also be obtained through the calculation of the formula, and the $\mathrm{V}$ value can also reflect the change of the vowel pattern more intuitively through specific numerical values. The details are shown in Table 1.

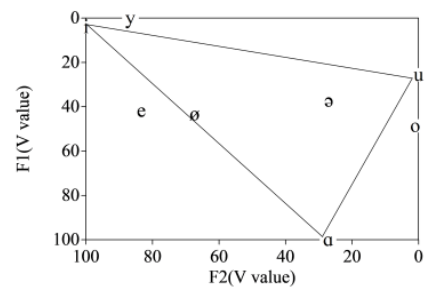

Figure 4 The pattern of vowels at the beginning of a word

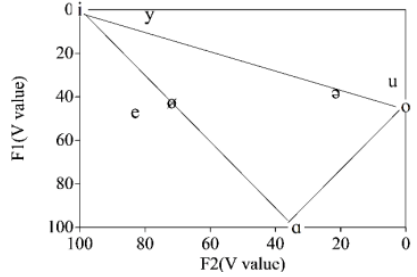

Figure 5 The pattern of vowels in a word

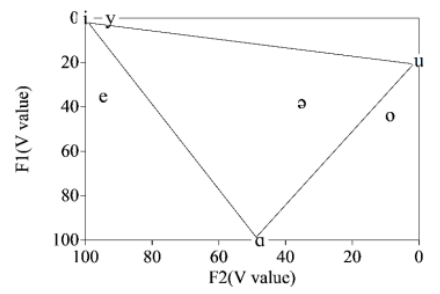

Figure 6 The pattern of ending vowels

Table $1 \mathrm{~V}$ value statistics

\begin{tabular}{|c|c|c|c|c|c|c|}
\hline \multirow[t]{2}{*}{ vowel } & \multicolumn{2}{|c|}{ Word beginning } & \multicolumn{2}{|c|}{ word middle } & \multicolumn{2}{|c|}{ word ending } \\
\hline & V2 & V2 & V1 & V2 & $\mathrm{V} 1$ & V2 \\
\hline $\mathbf{a}$ & 100 & 27 & 100 & 34 & 100 & 48 \\
\hline$\varnothing$ & 40 & 67 & 42 & 69 & 无 & 无 \\
\hline ə & 37 & 27 & 38 & 21 & 38 & 35 \\
\hline e & 42 & 83 & 47 & 83 & 35 & 95 \\
\hline $\mathrm{i}$ & 4 & 100 & 0 & 100 & 0.2 & 100 \\
\hline o & 49 & 1 & 44 & 0 & 44 & 9 \\
\hline $\mathrm{u}$ & 26 & 0 & 33 & 4 & 19 & 0 \\
\hline$y$ & 0 & 87 & 2 & 79 & 0 & 92 \\
\hline
\end{tabular}

From the above three pattern diagrams, the distribution of vowels in a word is a bit more concentrated than the distribution of vowels in the beginning and the word, and [i] and [a] both occupy the highest and lowest positions. In Figure 2.4 and Figure 6, the vertex vowels are composed of [i], [a], [u], and the vertex vowels in Figure 5 are composed of [i], [a], [o]. The following will discuss in detail:

(1) [a] is in the lowest position in the three vowel pattern diagrams. This can also be explained by combining Table 1 . The (V1) value of [a] is 100 at the beginning, middle, and end of the word. In terms of the front and back dimensions, the (V2) of [a] are: 27, 34, and 48 in order. Therefore, [a] cannot be directly defined as a complete low vowel, it can only show that [a] is in the middle and rear position;

[i] is in the forefront position in the three vowel patterns. Table 1 shows that the value of [i](V2) is 100 at the beginning, middle, and end of the word, and it is also at the highest position in the vowel pattern. , (V1) values are: $4,0,0.2$, so it can be explained that $[\mathrm{i}]$ is a high vowel; 
$[\mathrm{u}]$ is at the bottom in the vowel pattern of the beginning and end of the word, and the value of (V2) is 0 . Although there are some differences in the vowel pattern of the word, the value of (V2) is still very low. It is 4 , indicating that $[\mathrm{u}]$ belongs to the back vowel. In terms of high and low dimensions, $[\mathrm{u}]$ is next to high [i] and $\mathrm{y}$, which shows that $[\mathrm{u}]$ also belongs to the range of high vowels.

[o] It can be seen from Table 1 that the value of (V2) is 1,0 , and 9 in order. [O] is also at the back in the vowel pattern diagram. So it can be explained that [o] belongs to the back vowel.

(2) The difference in the non-vertex vowels $[\mathrm{y}],[\mathrm{e}],[\varnothing]$, and [ə] is quite obvious. Among them, [y] is relatively stable in terms of high and low dimensions. In the vowel pattern diagram, it is at the highest point except for [i], and the value of (V1) is $0,2,0$, which also shows that $[\mathrm{y}]$ belongs to high vowels. In terms of front and back dimensions, [y] is slightly behind in the pattern of vowels at the beginning of a word and the pattern of vowels in a word, but it is still in a close position as a whole. This can also be illustrated from Table 1, [y] the (V2) values of is: $87,79,92$, which are close to [i] and belong to the range of front vowels. Comparing [y] and [e], we can find that the tongue position of $[\mathrm{e}]$ is a bit forward than the tongue position of $[y]$.

For the distribution pattern of [ø], just refer to Figure 4 and Figure 5. The tongue position of [e] is higher than that of $[\varnothing]$. From the overall point of view, the vowel [ə ] is at the back position. From Table 1, the V2 value of the [ə] ]is generally smaller, in order: $27,21,35$.

\section{CONCLUSION}

Through the method of phonetic experiment, using the concept of phonetic pattern to compare and discuss the vowel phoneme map and vowel pattern map of the 8 vowels of Western Yugur language, the following will be summarized:

(1) From the vowel phoneme map, [i] and [y] are both at the lowest, and F1 has the smallest value, indicating that [i] and [y] have the highest tongue position when they are pronounced, and they are high vowels. At the end of the word [y], the vowel F2 has a larger span, and the tongue position is shifted backward. The scope of [a] has also changed. It changes vertically in the middle of the word and horizontally at the end of the word. [e] At the beginning and end of the word, we can see that it is closer to [i]. This is because [e] can be pronounced as [i] after the root consonants [g] and [k]. For example: [ $\mathrm{e}^{\mathrm{h}} \mathrm{rgek}$ can be pronounced as [ $\mathrm{e}^{\mathrm{h}} \mathrm{rgik}$ ], and [keyər] can be pronounced read as [kiyər], indicating that $[\mathrm{e}]$ has allophone variants. ${ }^{[6]}$ In the vowel phoneme map, [o], [u] and the vowel [ə], the vowels in the beginning of the word and in the word are more concentrated, and there is a phenomenon of mutual overlap. There are also [i] and [y], [ø] and [e], there are also some overlaps, indicating that these vowels are pronounced similarly in some syllables, probably because $[\varnothing]$ and $[\mathrm{e}]$ are both the front low vowels, [o], $[\mathrm{u}]$, and [ə], the vowels are positioned at the back, all belong to the back vowels.

(2)In the vowel pattern diagram, you can clearly see the changes of each vowel. [a] is always a low vowel, but F2 is changing. When in a word, F2 is lowered, indicating that the tongue is moving backward. This is because [a] is in the [G], [q], and When [h] and [y] are spelled together, the tongue of $\mathrm{a}$ is clearly behind. ${ }^{[7]}$

(3)In the vowel phoneme map, it can be found that there are overlaps between vowels. In the vowel pattern map, these two vowels are relatively close. It is judged that these two vowels have some similarities in the pronunciation of certain syllables. This phenomenon of inconsistent pronunciation of the same word without any grammatical changes is called free replacement of phonetics in linguistics. ${ }^{[8]}$ It is not difficult to understand that in the Western Yugur language, some vowels overlap during the phonetic experiment, which also confirms that there is free phonetic substitution in the Western Yugur language.

\section{ACKNOWLEDGMENTS}

This research was funded and completed by the Northwest Minzu University 2021 Central University' s Basic Scientific Research Project Funding Special Funds for Postgraduate Projects (Project Number: Yxm2021071 Project Name: Experimental Research on the Acoustic Features of Western Yugur Language Vowels).Also are the Key Laboratory of China's Ethnic Languages and Information Technology ( Northwest Minzu University), Ministry of Education.

\section{REFERENCES}

[1] Zhong Jinwen. The regional characteristics of the unique ethnic language and culture in Ganqing and Qinghai [M]. Beijing: Central University for Nationalities Press, 2007.

[2] Zhong Jinwen (Editor-in-Chief). Collected Works of Yugur Studies Abroad [C]. Beijing: Central University for Nationalities Press, 2008.

[3] Lei Xuanchun. Western Yugur Chinese Dictionary [M]. Sichuan: Sichuan Nationalities Publishing House, 1992.

[4] Shi Xiujuan. The vowel pattern of Chinese dialects[M]. Beijing: China Social Sciences Press, 2010. 
[5] Shi Feng. Phonetic pattern - the intersection of phonetics and phonology [M]. Beijing: The Commercial Press, 2008.

[6] Zhong Jinwen. Collection of Studies on Yugur Nationality in China [M]. Beijing: Nationalities Publishing House, 2002.
[7] Zhong Jinwen. Collected Works of Yugur Studies Abroad[M]. Beijing: Central University for Nationalities Press, 2008.

[8] Zhong Jinwen. A Study on the Description of Yugur Language in the West $[\mathrm{M}]$. Beijing: National Publishing House, 2009. 